\title{
Sternohyoid Muscles for Reconstruction After Thyroid Cartilage Anterior Partial Resection
}

\author{
Anna Pamela Dela Cruz, MD; Marc Remacle, MD, PhD; Jerôme Keghian, MD; \\ Pierre Lepage, MD; Florence Rogister, MD
}

\section{INTRODUCTION}

Defects in the thyroid cartilage following certain conditions such as chondronecrosis, trauma, or laryngeal cancer surgery mandate a form of reconstruction to maintain its physiologic function. In this article, we present the management of two complicated cases requiring anterior thyroid cartilage partial resection and reconstruction.

\section{METHODS}

\section{Technique}

Following the neck incision and development of the subplatysmal flaps, the infrahyoid muscles were retracted laterally to expose the thyroid cartilage. Anterior partial resection of the thyroid cartilage and smoothening of the edges of the remaining thyroid ala was performed using the micromotor drill and burrs. Approximation of remaining thyroid ala was done using resorbable sutures. The sternohyoid muscles were then stretched onto the area of the defect and apposed using resorbable sutures (Fig. 1).

\section{RESULTS}

\section{Case 1}

This is a case of a 54-year-old female presenting with necrosis of the thyroid cartilage secondary to uncontrolled diabetes. The patient consulted following microsurgery for Reinke's edema and subsequent sessions of speech therapy. According to the patient, the said treatment was insufficient to improve her voice. Upon examination, a bilateral vocal fold scar was seen on endoscopy. Furthermore, there was also a glottal gap and vocal fold atrophy noted. A

From the Department of Otorhinolaryngology (A.P.D.), Philippine General Hospital, Manila, the Philippines; and the Department of Otorhinolaryngology-Head and Neck Surgery (M.R., J.K., P.L., F.R.), Centre Hospitalier de Luxembourg-Eich, Luxembourg City, Luxembourg.

Editor's Note: This Manuscript was accepted for publication February 13, 2017.

The authors have no funding, financial relationships, or conflicts of interest to disclose.

Send correspondence to Anna Pamela Dela Cruz, MD, Department of Otorhinolaryngology, Philippine General Hospital, Taft Avenue, Ermita, Manila, Philippines. E-mail: pamelacdelacruz@gmail.com

DOI: 10.1002/lary.26575 bilateral Isshiki type I medialization thyroplasty ${ }^{1}$ using the Montgomery implant system ${ }^{2}$ was then proposed and undertaken. The patient was discharged the day after the procedure; however, after 10 days, she presented with purulent coughing. Endoscopy revealed a superinfection with purulent secretion, granuloma formation, and extrusion of the implant on the left vocal fold (Fig. 2). The patient had diabetes mellitus, but was not compliant with medications, which may have been a contributory factor to the development of a major superinfection and consequently implant extrusion. Surgical exploration to remove the prosthesis on the left vocal fold was done. On exploration, there was considerable necrosis of the thyroid cartilage, and the vocal folds were floating due to destruction of its attachment anteriorly to the inner surface of the thyroid cartilage (Fig. 3).

The necrotic part of the thyroid ala was resected to obtain two sharp, regular edges, leaving a gap anteriorly. To secure the position of the vocal folds, it was sutured together with the remnant cricothyroid ligament onto the cricoid cartilage. The sternohyoid muscles were stretched onto the open larynx and sutured together with five stitches of Vicryl 3-0. The neck was cleaned and the skin closed. The patient was not tracheotomized and was extubated the following day. Antibiotic therapy using metronidazole was given for 7 days. There was no airway problem postoperatively. The voice recovered progressively with the healing; however, the left vocal fold was affected by a major atrophy and scarring process. After 2 months, injection laryngoplasty was undertaken to further improve the voice. The patient had a total of four injections on the left vocal fold using collagen and hyaluronic acid.

After 1 year, a buccal mucosa free graft was performed to address the scar. On follow-up after 7 months, the left vocal fold was noted to have good mucosalization and the patient had a more satisfactory speech outcome.

\section{Case 2}

This is a case of a 53-year-old male presenting with recurrent glottic carcinoma. The patient consulted for evaluation of a possible tumor recurrence in the vocal 

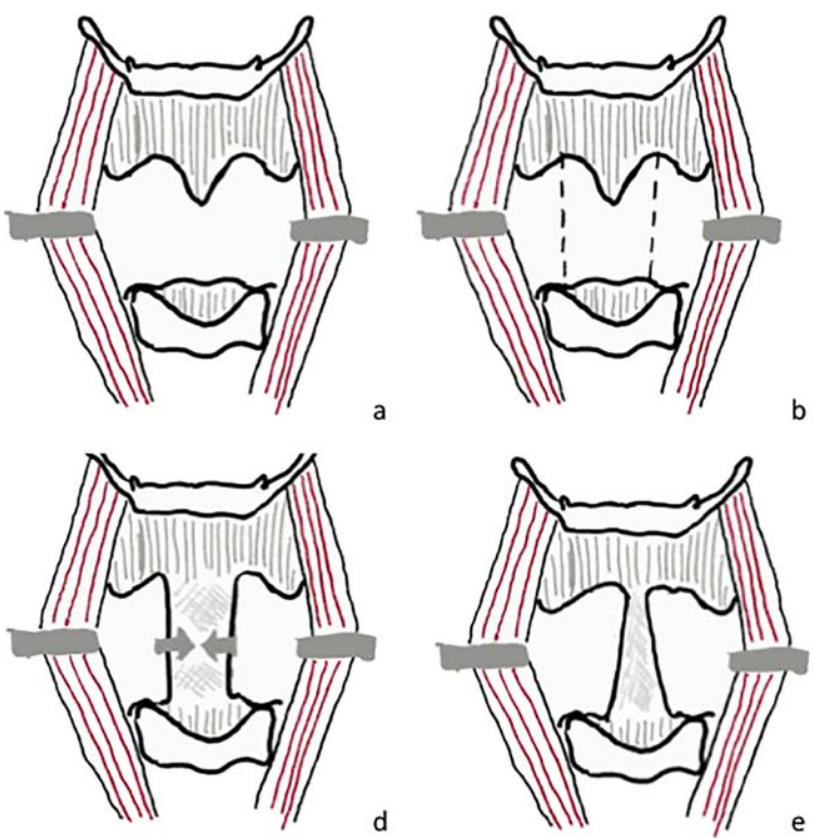

fold following primary radiotherapy and type I cordectomy. Regular endoscopy was unremarkable for 1 year and 10 months until an erythema on the midinferior portion of the epiglottis and anterior commissure was noted (Fig. 4). Computed tomography was requested that revealed minimal extension of the lesion to the pre-epiglottic space. There was also a palpable cervical lymph node on the right, thus staging the patient as stage III rT3N1M0.

The patient underwent a transoral $\mathrm{CO}_{2}$ laser microsurgery resection. Tumor resection included the anterior commissure, anterior one-third of the bilateral true vocal folds, midinferior portion of the epiglottis, pre-epiglottic space, down to the level of the thyroid cartilage and inferior border of the cricoid cartilage (Fig. 5), thus a type VI cordectomy according to the European Laryngological Society classification of endoscopic cordectomy. ${ }^{3}$

Of particular significance in this case was the tumor adherence at the anterior commissure, cricothyroid ligament, and adjacent inner surface of the thyroid cartilage. An external approach for thyroid cartilage

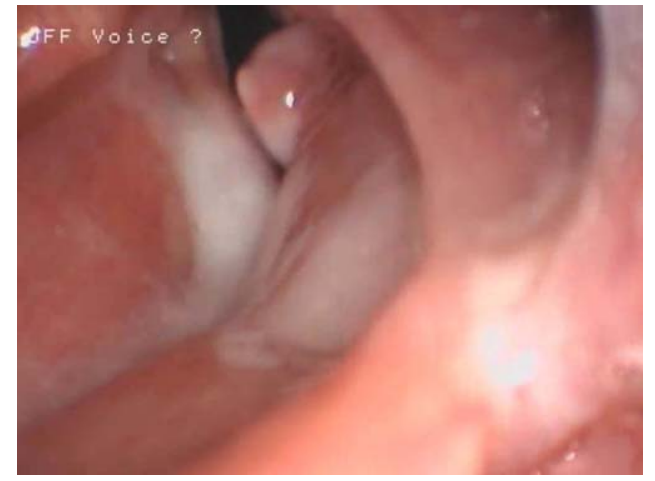

Fig. 2. Indirect laryngoscopy showing implant extrusion on the left vocal fold. [Color figure can be viewed in the online issue, which is available at www.laryngoscope.com.]
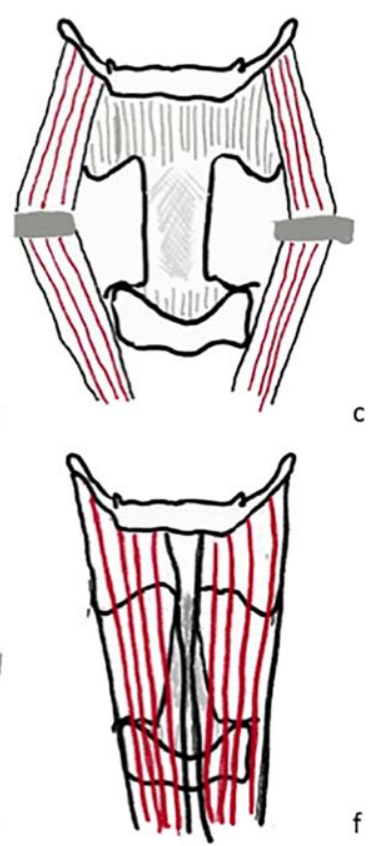

Fig. 1. Diagram of the external approach for anterior partial resection of the thyroid cartilage and reconstruction using the sternohyoid muscles. [Color figure can be $f$ viewed in the online issue, which is available at www.laryngoscope.com.]

resection was then decided to ensure an oncologically safe margin. Following the neck dissection (levels II, III, IV) on the right, the infrahyoid muscles were retracted to expose the thyroid cartilage. The anterior portion of the thyroid cartilage where tumor adherence was noted was resected (Fig. 6A). The remaining ala on each side of the thyroid cartilage was approximated using Vicryl sutures. The sternohyoid muscles were stretched onto the area where the thyroid cartilage ala was fused and sutured together (figure 6B). Fibrin glue was also applied to reinforce the closure. All margins sent for frozen section were negative for tumor. Following reconstruction, closure of neck incision was done in layers.

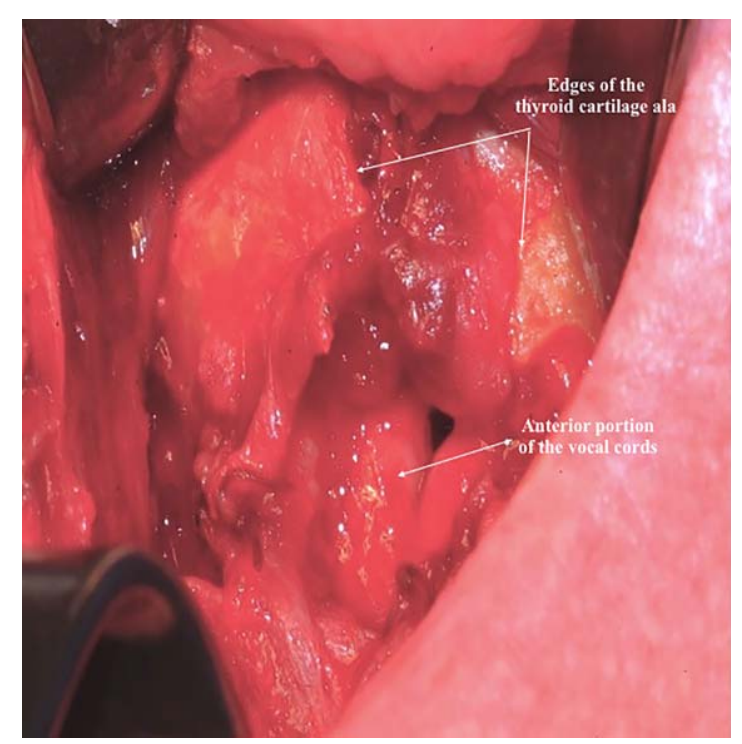

Fig. 3. Intraoperative findings showing necrosis of the thyroid cartilage. [Color figure can be viewed in the online issue, which is available at www.laryngoscope.com.] 


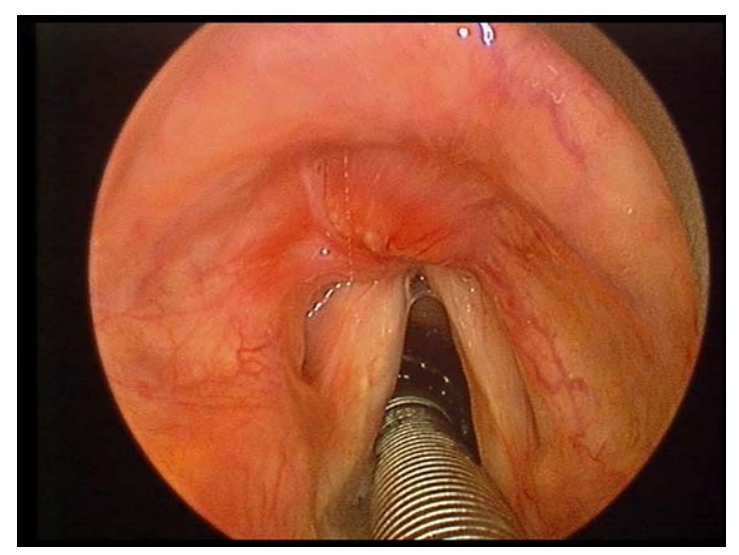

Fig. 4. Direct laryngoscopy showing the area of recurrence on the inferior portion of the laryngeal surface of the epiglottis and anterior commissure. [Color figure can be viewed in the online issue, which is available at www.laryngoscope.com.]

The patient was not tracheotomized, and delayed extubation was done 24 hours postsurgery. The patient was able to swallow liquids and solids 2 days after surgery and discharged after 4 days without complications. Two weeks postoperatively, there was note of good cicatrization on endoscopy. One month postoperatively, endoscopy showed good healing. Final histopathology report revealed a moderately differentiated squamous cell carcinoma. All areas of resection were negative for tumor.

\section{DISCUSSION}

The first case presents a very challenging situation following surgery for a benign condition of the larynx. Following medialization thyroplasty for a scarred larynx with suboptimal voice outcome, the patient had implant extrusion and chondronecrosis of the thyroid cartilage. In literature, implant migration is one of the complications of medialization thyroplasty, although in a very small percentage of cases. ${ }^{4,5}$ After a thorough literature search of cases presenting with chondronecrosis after thyroplasty, no similar article was found. In this case, one plausible cause for such complication would be the

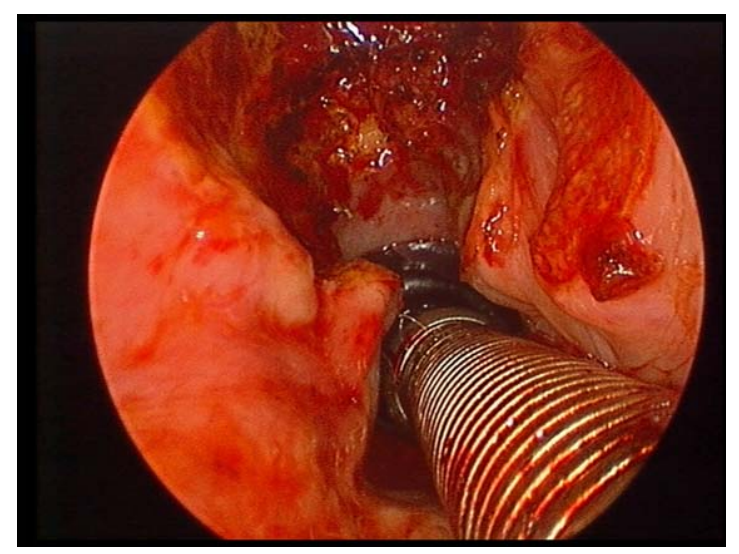

Fig. 5. Postoperative view showing the extent of resection. [Color figure can be viewed in the online issue, which is available at www.laryngoscope.com.]
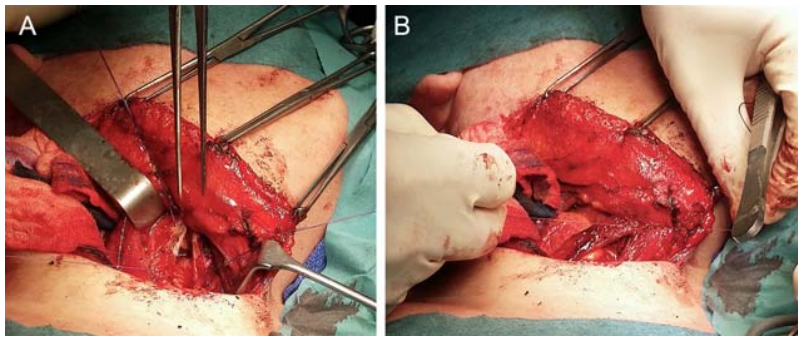

Fig. 6. External approach. (A) Anterior partial resection of the thyroid cartilage. (B) Reconstruction using the sternohyoid muscles. [Color figure can be viewed in the online issue, which is available at www.laryngoscope.com.]

uncontrolled diabetes. Studies have shown that for this metabolic disorder, there is a decrease in chemotaxis of inflammatory effector cells into the wound that should engulf invading bacteria and promote removal of necrotic foci. In addition, there is also an increase in the generation of proinflammatory cytokines such as tumor necrosis factor- $\alpha$ and matrix metalloproteinases, resulting in impedance in the phases of wound healing and wound closure. ${ }^{6}$ In our patient, following the removal of the necrotic thyroid cartilage, a significant defect was left anteriorly. Reconstruction was done by simply using the sternohyoid muscles onto the open larynx.

The second case presents some of the controversies in management of recurrent glottic cancer. First is the therapeutic decision on recurrent glottic carcinoma after failed radiotherapy, and second is treatment of anterior commissure involvement. The anterior commissure, being close to the thyroid cartilage, mandates a preoperative evaluation with computed tomography or magnetic resonance imaging, because tumor extension into the thyroid cartilage easily upstages the patient. Open partial laryngectomy and transoral laser microsurgery are the treatment modalities available for lesions involving the anterior commissure. In several reports, transoral laser microsurgery has been the preferred treatment because of its advantages including shorter duration of hospitalization, avoidance of tracheostomy, and better voice quality. ${ }^{7,8}$ Some reports, however, had concerns on exposure and adequacy of resection using an endoscopic approach. To address the issue on adequate resection without compromising too much of the laryngeal function, Shapshay et al. proposed a combined endoscopic and external approach for glottic cancer involving the anterior commissure. Following endoscopic excision, an external approach is performed by creating a window in the thyroid cartilage and removing the specimen en bloc. Glottic reconstruction is then carried out using the sternohyoid muscle flap by either a bipedicled muscle flap with overlying skin or a unipedicled muscle flap with a graft of free mucosa. ${ }^{9-11}$ The use of sternohyoid muscles earlier described by Ogura and Biller in 1969 following frontolateral hemilaryngectomy is a simple technique, which has been used for laryngeal reconstruction for over several decades. ${ }^{12}$ The approach has also been modified by other surgeons and still proves to be a reliable technique for reconstruction. In our patient, a technique 
of combined transoral laser microsurgery and external approach was utilized. Complete tumor resection was done endoscopically, but an external approach was also performed due to tumor adherence at the anterior commissure and cricothyroid ligament. Instead of just creating a window, anterior partial resection of the thyroid cartilage was done, leaving a gap anteriorly. Reconstruction was completed by apposition of the remaining thyroid cartilage ala and suturing the sternohyoid muscles for closure of the defect.

Reconstruction of defects following surgical extirpation is important to maintain the physiologic function of the larynx. For both cases presented, reconstruction using sternohyoid muscles after anterior partial resection of the thyroid cartilage were successful. Even if onethird or more of the thyroid cartilage ala were removed, closure using the sternohyoid muscles was adequate and efficient. In both patients, the procedure was done without the need of a tracheotomy, and there were no airway problems encountered postoperatively. Speech and swallowing outcomes were also satisfactory for both patients.

\section{CONCLUSION}

These cases illustrate that the use of sternohyoid muscles can be a simple, effective, and reliable approach for reconstruction following anterior partial resection of the thyroid cartilage for benign and malignant conditions of the larynx.

\section{BIBLIOGRAPHY}

1. Isshiki N. Phonosurgery-Theory and Practice. Tokyo, Japan: Springer; 1989:82-109

2. Montgomery W, Montgomery SK. Montgomery thyroplasty implant system. Ann Otol Rhinol Laryngol Suppl 1997;170:1-16.

3. Remacle M, Van Haverbeke C, Eckel H, et al. Proposal for revision of the European Laryngological Society classification of endoscopic cordectomies. Eur Arch Otorhinolaryngol 2007;264:499-504.

4. Rosen CA. Complications of phonosurgery: results of a national survey. Laryngoscope 1998;108(11 pt 1):1697-1703.

5. Young VN, Zullo TG, Rosen CA. Analysis of laryngeal framework surgery: 10-year follow-up to a national survey. Laryngoscope 2010;120: $1602-1608$.

6. Goova MT, Li J, Kislinger T, et al. Blockade of receptor for advanced glycation end-products restores effective wound healing in diabetic mice. Am J Pathol 2001;159:513-525.

7. Steiner W, Ambrosch P, Rödel RM, Kron M. Impact of anterior commissure involvement on local control of early glottic carcinoma treated by laser microresection. Laryngoscope 2004;114:1485-1491.

8. Rödel RM, Steiner W, Müller RM, Kron M, Matthias C. Endoscopic laser surgery of early glottic cancer: involvement of the anterior commissure. Head Neck 2009;31:583-592.

9. Shapshay SM, Wang Z, Rebeiz EE, et al. "Window" laryngoplasty: a new combined laser endoscopic and open technique for conservation surgery. Ann Otol Rhinol Laryngol 1994;103:679-685.

10. Shapshay SM, Wang Z, Rebeiz EE, Perrault DF, Pankratov MM. A combined endoscopic $\mathrm{CO}_{2}$ laser and external approach for treatment of glottic cancer involving the anterior commissure: an animal study. Laryngoscope 1996;106:273-279.

11. Rebeiz EE, Wang Z, Annino DJ, McGilligan JA, Shapshay SM. Preliminary clinical results of window partial laryngectomy: a combined endoscopic and open technique. Ann Otol Rhinol Laryngol 2000;109:123-127.

12. Ogura JH, Biller HF. Glottic reconstruction following extended frontolateral hemilaryngectomy. Laryngoscope 1969;79:2181-2184. 